\title{
Investigation of cross flow over a circular cylinder at low Re using the Immersed Boundary Method (IBM)
}

\author{
K. Rehman \\ Department of Mechanical Engineering, \\ College of Electrical and Mechanical Engineering, \\ National University of Sciences and Technology, Islamabad, Pakistan
}

\begin{abstract}
Flow over a circular cylinder is laminar at $\mathrm{Re}=40$. At $\mathrm{Re}=100$ distinct vortex shedding can be observed. In this paper, a fully implicit method with the fractional step has been used to discretize the Navier-Stokes equations. The cylinder boundary condition is imposed using the Immersed Boundary Method (IBM). IBM is a relatively new technique where Cartesian mesh is employed effectively for complex geometries. The method employs second-order linear and bilinear interpolations for calculation of forcing velocities which are used to enforce the no slip boundary conditions over the virtual boundary of the circular cylinder. The statistics like mean $C_{D}$, rms $C_{L}$, and Strouhal number are calculated using inverse distance interpolation of field variables over the virtual boundary of the cylinder. Other plots like streamlines and vorticity are also included. Good agreement of results with experimental and numerical simulation data is observed.

Keywords: Immersed Boundary Method, bilinear interpolation, forcing velocities.
\end{abstract}

\section{Introduction}

Flow past a circular cylinder is one of the most studied phenomena in fluid dynamics. However, still much is to be learned about the complexities of this simple flow. In this paper, an effort is made to accurately investigate the flow past a circular cylinder at different Re. The object geometry placed in the flow has been realized using IBM technique. IBM is a relatively new technique, in which forcing velocities are applied inside the virtual solid body near the 
boundary to enforce the boundary conditions. The method uses a staggered Cartesian grid which is easy and computationally cheap to work with. Kim et al. [1] proposed a fully implicit splitting type fractional step method (FSM) technique. Crank-Nicholson discretization is applied on both the diffusion and convection terms. Discretized equations are arranged in matrix form with momentum boundary condition $(\mathrm{mbc})$ and continuity boundary conditions (cbc) matrices. The velocity and pressure decoupling by LU decomposition allows the direct solution of equations resulting in lesser computations. Direct calculation of pressure from Poisson equation results in an efficient algorithm. In this work, the method of Kim et al. [2] is employed where the boundary conditions are implemented using IBM on a staggered Cartesian grid. The method is applied to flow over a cylinder in laminar regime to validate its effectiveness at a low $\mathrm{Re}=40$. In addition validation at $\mathrm{Re} 100$ is also done. Comparison with published numerical and experimental results is done. The results presented are comparable to earlier studies. The statistics like mean $C_{D}$, rms value of $C_{L}$, and Strouhal number are calculated using inverse distance interpolation of field variables over the virtual boundary of the cylinder. The IBM and monitoring of the statistics is done internally over the time history for each solution iteration.

\section{Numerical procedure}

The DNS code developed using fully implicit fractional step method and IBM technique has been used to calculate the flow field variables over the body. IBM was first presented by Peskin in 1972 to simulate cardiac mechanism and associated blood flow. Here the formulation given by Kim et al. [2] with finite volume method using both momentum forcing and mass source terms is employed. Staggered Cartesian grid is employed to solve the equations with time step corresponding to CFL 1.2 which is well within the stability limits investigated for $\mathrm{Re}=40$ regime of flow over a cylinder. For $\mathrm{Re}=100$ a CFL value of 0.75 was used. The unsteady incompressible Navier-Stokes equations in the index notation are:

$$
\begin{aligned}
\frac{\partial u_{i}}{\partial t}+u_{j} \frac{\partial u_{i}}{\mathrm{u}_{\mathrm{j}}} & =-\frac{1}{\rho} \frac{\partial p}{\partial x_{i}}+v \frac{\partial^{2} \mathrm{u}_{\mathrm{i}}}{\partial^{2} x_{j} x_{j}} \\
\frac{\partial u_{i}}{\partial x_{i}} & =0
\end{aligned}
$$

Here the pressure is coupling with in momentum equations. After spatial and temporal discretization, the momentum equation becomes:

$$
\frac{u_{i}^{n+1}-u_{i}^{n}}{\Delta t}=\frac{1}{R e} \frac{\delta^{2} u_{i}^{n+1}}{\delta x_{j} x_{j}}-u_{i}^{n+1} \frac{\delta u_{i}^{n+1}}{\delta x_{j}}-\frac{\partial p^{n+1}}{\partial x_{i}}
$$

Pressure is taken out and equation is solved for the intermediate velocity $\tilde{u}_{l}$. This velocity does not satisfy incompressibility condition.

$$
\frac{\widetilde{u_{l}}-u_{i}^{n}}{\Delta t}=\frac{1}{R e} \frac{\delta^{2} \widetilde{u_{l}}}{\delta x_{j} x_{j}}-\widetilde{u_{l}} \frac{\delta \widetilde{u_{l}}}{\delta x_{j}}
$$


Pressure is calculated using the Poisson equation, which would ensure a divergence free velocity field at next time level

$$
\frac{1}{\Delta t} \frac{\delta \widetilde{u_{l}}}{\partial x_{i}}=\frac{\partial^{2} p^{n+1}}{\partial x_{i} \partial x_{i}}
$$

The velocity field at a new time level is calculated using

$$
\frac{u_{i}^{n+1}-\widetilde{u_{l}}}{\Delta t}=-\frac{\partial p^{n+1}}{\partial x_{i}}
$$

For the IBM implementation, a forcing term ' $\mathrm{f}_{\mathrm{i}}$ ' is added on the left hand side of momentum equation and is evaluated over the ghost control volumes from which the forcing velocities are calculated to ensure no slip boundary condition over the cylinder virtual surface. A source term ' $\mathrm{q}$ ' is added into the continuity equation to preserve mass continuity over the ghost control volumes. The detailed procedure implemented is as suggested by Kim et al. [2]. The evaluation of statistics like $C_{D}$ and $C_{L}$ requires evaluation of flow field variables like velocities, pressure and gradients over the virtual surface of the cylinder. The dimensionless drag coefficient is defined by:

$$
C_{D}=\frac{F_{D}}{(1 / 2) \rho u_{\infty}^{2} D}
$$

where $F_{D}$ is the drag force of the cylinder in the direction of free stream velocity and $\mathrm{D}$ is the cylinder diameter used as the reference length. The lift coefficient is given by:

$$
C_{L}=\frac{F_{L}}{(1 / 2) \rho u_{\infty}^{2} D}
$$

The method of calculation of forces on a body in fluid flow is given by Lai and Peskin [13]. The integral form of the x-component of the momentum equations on any fluid domain $\Omega$ o can be described by:

$$
\begin{gathered}
\frac{\partial}{\partial t} \int_{\Omega o} \rho u_{1} d \boldsymbol{x}+\int_{\partial \Omega o} \rho u_{1} \boldsymbol{u} \cdot \boldsymbol{n} d s= \\
-\int_{\Omega o} p n_{1} d \boldsymbol{x}+\int_{\partial \Omega o} \mu\left(\frac{\partial u_{i}}{\partial x_{j}}+\frac{\partial u_{j}}{\partial x_{1}}\right) n_{j} d \boldsymbol{s}+\int_{\Omega o} f_{1} d \boldsymbol{x}
\end{gathered}
$$

When the flow is steady we may write

$$
\int_{\partial \Omega o} \rho u_{1} \boldsymbol{u} . \boldsymbol{n} d s=-\int_{\Omega o} p n_{1} d \boldsymbol{x}+\int_{\partial \Omega o} \mu\left(\frac{\partial u_{i}}{\partial x_{j}}+\frac{\partial u_{j}}{\partial x_{1}}\right) n_{j} d \boldsymbol{s}+\int_{\Omega o} f_{1} d \boldsymbol{x}
$$

The drag force can thus be evaluate as

$$
F_{D}=-\int_{\partial \Omega o} \rho u_{1} \boldsymbol{u} \cdot \boldsymbol{n} d s-\int_{\Omega o} p n_{1} d \boldsymbol{x}+\int_{\partial \Omega o} \mu\left(\frac{\partial u_{i}}{\partial x_{j}}+\frac{\partial u_{j}}{\partial x_{1}}\right) n_{j} d \boldsymbol{s}
$$


In order to evaluate this line integral, a second order inverse distance interpolation is employed to calculate the flow variables. The second order accurate interpolation ensures the overall second order accuracy of the method.

\section{Computational details}

For the $\operatorname{Re} 40$ and 100 simulations the computational domain consists of rectangular domain of $100 \mathrm{~d} \times 70 \mathrm{~d} \times 4 \mathrm{~d}$ where $\mathrm{d}=1$ is the diameter of the cylinder. The centre of the cylinder is placed at Xc $=40 \mathrm{~d}$ and $\mathrm{Yc}=35 \mathrm{~d}$. This is similar to the domain used and verified by Kim et al. [2] for Re 40 and 100 simulations. The grid points used are 2048 in $x, 512$ in y and 4 in z direction for $\operatorname{Re} 40$ and 4096 in $x, 512$ in $y$ and 4 in $\mathrm{z}$ for the Re 100 simulations. Clustering of points around the centre in y direction is done using sinh function. For Re 40 simulation there are 42 and 69 points distributed inside the cylinder along $\mathrm{x}$ and $\mathrm{y}$ direction respectively. The resulting time step is approximately $0.012 \mathrm{sec}$ and 0.005 respectively. The number of IBM control volumes comes out to be 104 and 126 respectively, where forcing velocities are being calculated. The domain is shown in figure 1. A portion of the domain with cylinder placed over the grid is shown in figure 2. It can be seen that the cylinder is very small as compared to the domain. The inlet uniform velocity is set as $U=1$ (constant) and the Re is used to set the corresponding viscosity. The far field is used on the top and bottom sides. Periodic b.c. is applied along the span. Outflow is set as convective boundary.

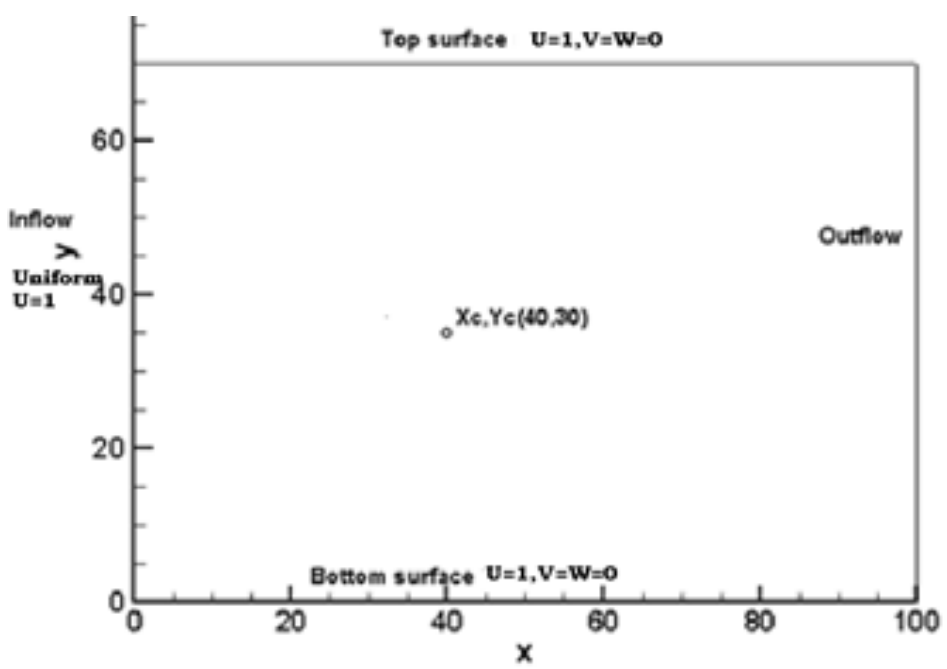

Figure 1: Computational domain used for Re 40 and 100. 


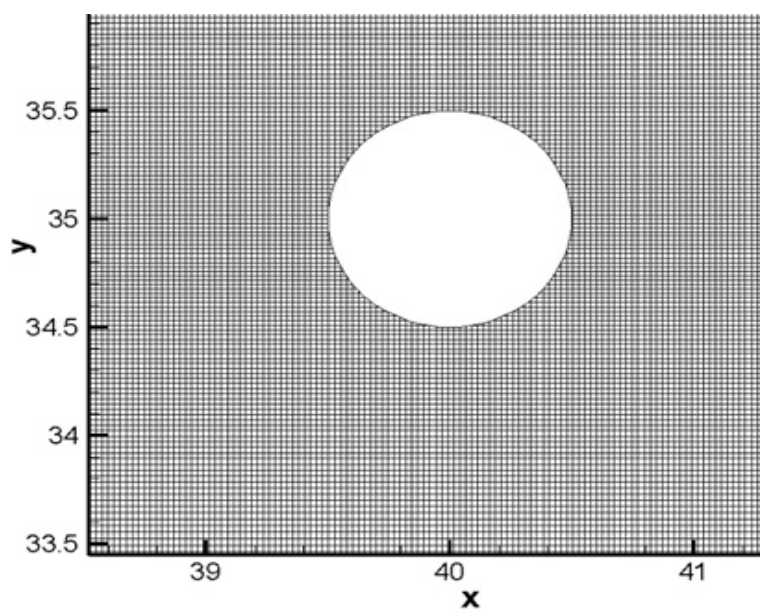

Figure 2: Portion of the grid around the cylinder.

\section{Results and discussion $\operatorname{Re} 40$}

The u-velocity field and the streamlines over the $\mathrm{u}$ velocity contours are presented in figure 3 and 4. It is seen that the far field is well set and sufficient region is available around the cylinder for development of flow. For the velocity contours seen in figure 3, the wake and front disturbed area are clearly seen. The streamlines are shown in figure 4 . The standing vortex at the rear is well defined. The length given as the ratio of standing vortex to the reference diameter of the cylinder $(\mathrm{Lw} / \mathrm{d})$ is seen to be 2.305 . This is in agreement with

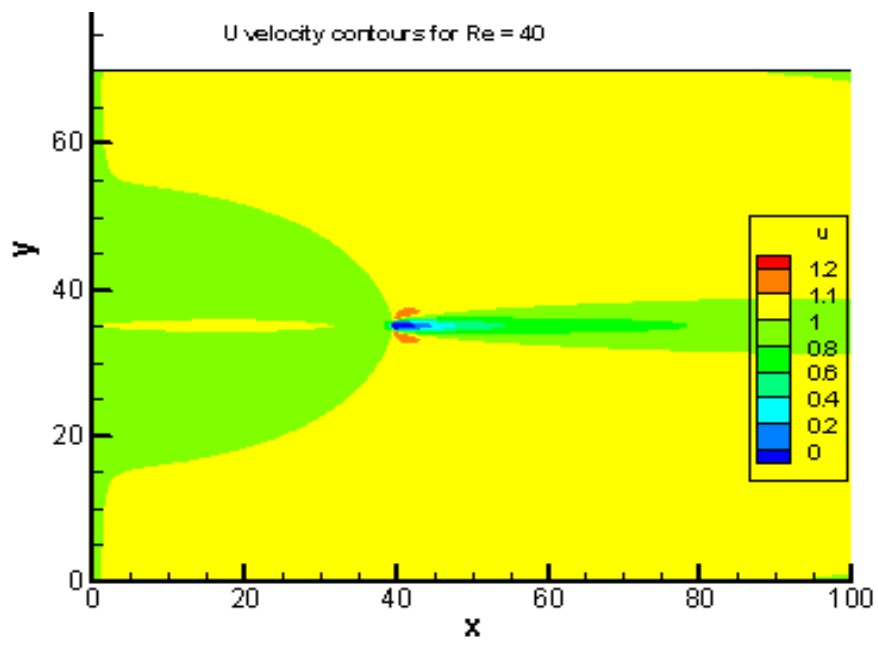

Figure 3: The U velocity field around the cylinder. 


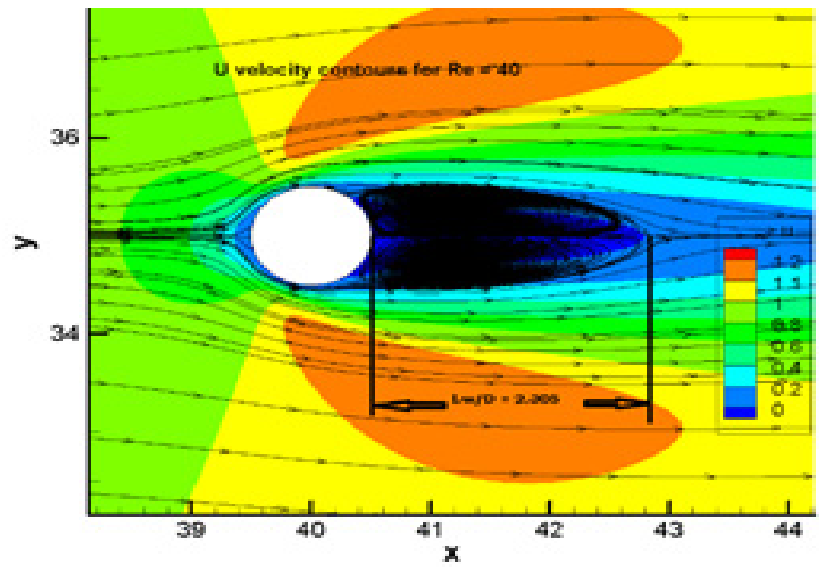

Figure 4: Standing vortices at the rear of the cylinder. Re 40.

earlier studies. The value of $\mathrm{C}_{\mathrm{D}}$, the drag coefficient based on reference length diameter of the cylinder, obtained after the flow was fully developed is 1.5515. The computational time corresponds to 105 seconds. A comparative table made with the data from different references is given in table 1.

\section{Results and discussion $\operatorname{Re} 100$}

The $\mathrm{u}$-velocity contours and the stream lines are presented in figures 5 and 6 respectively. The alternating vortex shedding is seen in these plots. Due to this the drag oscillations and alternating lift is occurring. The streamlines also

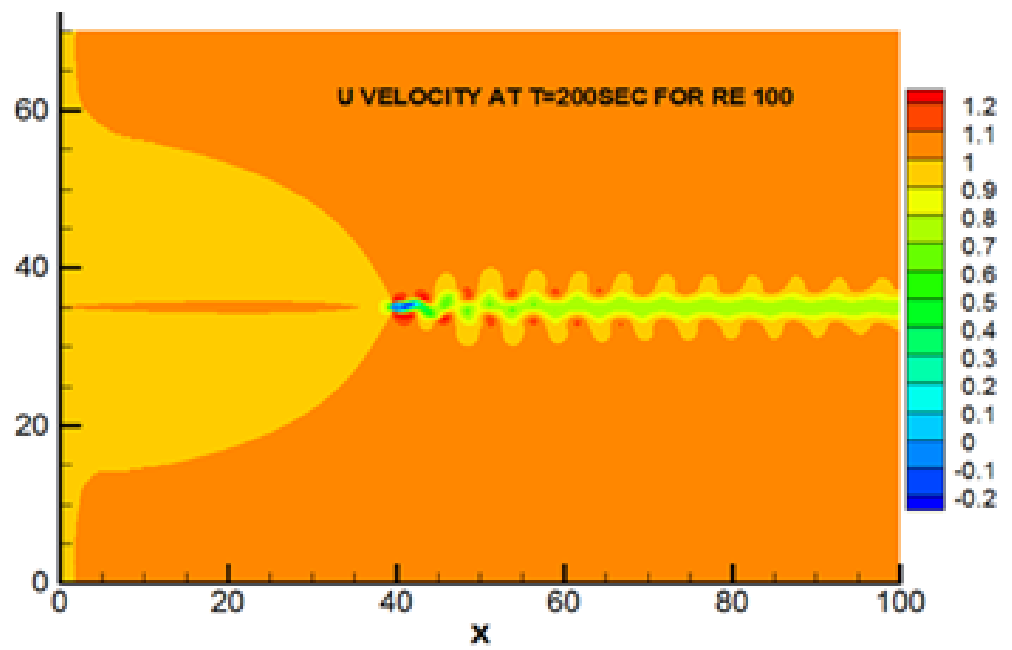

Figure 5: U velocity contours for Re 100 . 


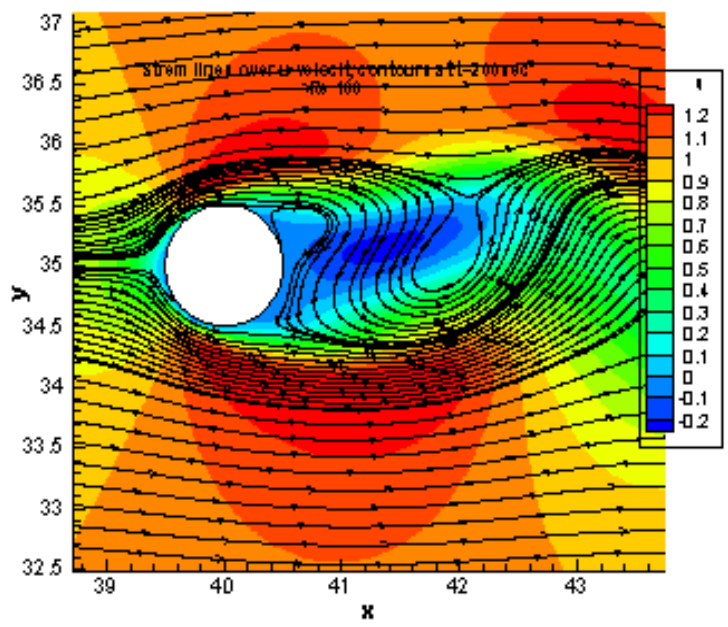

Figure 6: Stream lines around the cylinder for Re 100.

confirm the vortex shedding. The drag coefficient is plotted in figure 7 . The $\mathrm{Cd}$ mean value after 190 seconds of simulation time is 1.398 . The lift coefficient vs. time plot shows that the shedding was started after 100 seconds of simulation and was fully developed after 145 seconds (refer figure 8 ). The Strouhal number is seen to be 0.16 , which is in good agreement in comparison to Kim et al. [2] papers value of 0.165 and Shen et al. [12] estimation of 1.66.

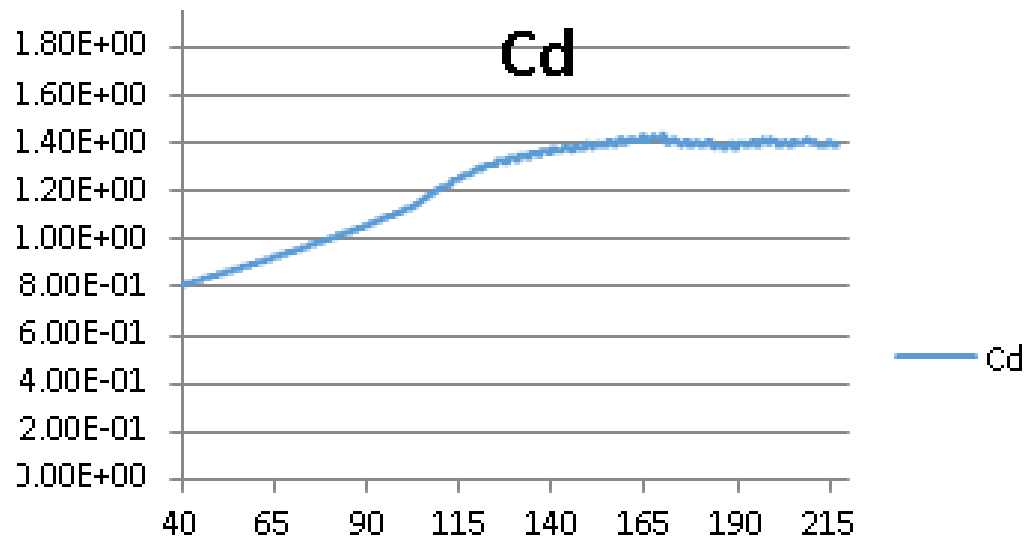

Figure 7: Cd vs. Time for Re 100. 


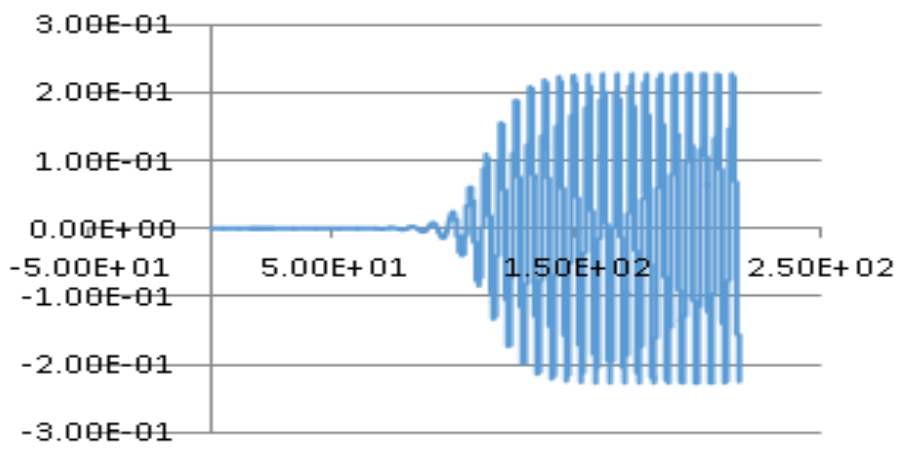

Figure 8: $\quad \mathrm{Cl}$ vs. Time for Re 100.

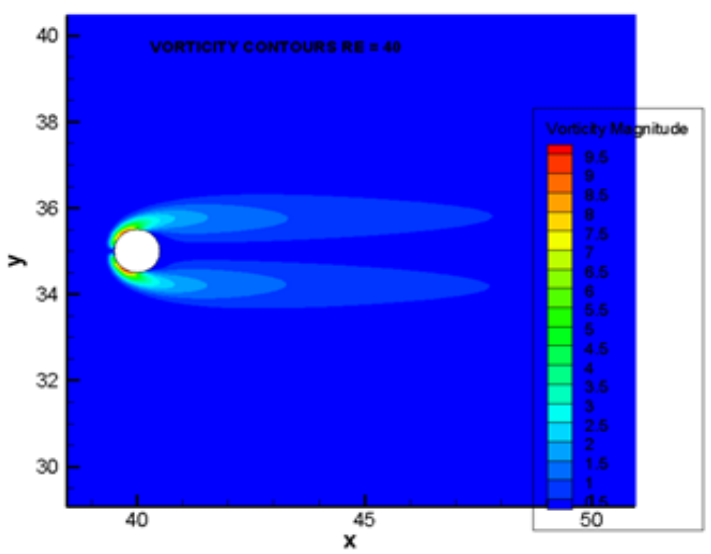

Figure 9: Vorticity plot for $\mathrm{Re}=40$.

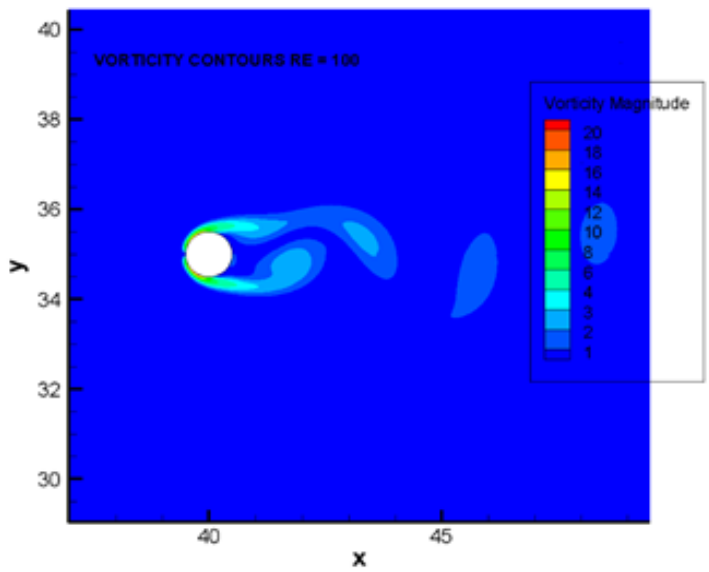

Figure 10: Vorticity plot for Re 100. 


\section{Conclusion}

The value of $C_{D}$ and $C_{L}$ based on reference length diameter of the cylinder, obtained after the flow was fully developed are given in comparison table 1 below. It is concluded that scheme is effective in predicting the flow field around a curved surface like cylinder. More simulations at high $\mathrm{Re}=3900$ and higher can be done in future. Turbulent flow structures can be studied in the wake of complex structures like circular cylinders.

Table 1: Comparison of values with earlier papers.

\begin{tabular}{|l|c|c|c|c|c|}
\hline Author & \multicolumn{2}{|c|}{ Re40 } & \multicolumn{3}{c|}{ Re 100 } \\
\hline & $\mathrm{C}_{\mathrm{d}}$ & $\mathrm{L}_{\mathrm{w}} / \mathrm{D}$ & $\mathrm{C}_{\mathrm{d}}$ & $\mathrm{St}$ & $\mathrm{C}_{\mathrm{l}}$ (rms) \\
\hline Present & 1.55 & 2.305 & 1.398 & 0.16 & 0.32 \\
\hline Lima et al.[3] & 1.54 & 2.45 & 1.39 & 0.164 & - \\
\hline Tseng and Ferziger [11] & 1.53 & 2.21 & 1.42 & 0.164 & 0.29 \\
\hline Ye et al.[10] & 1.52 & 2.27 & - & - & - \\
\hline Huang and Sung [9] & 1.56 & 2.27 & 1.37 & 0.167 & 0.33 \\
\hline Kim et al.[2] & 1.51 & - & 1.33 & 0.165 & 0.32 \\
\hline Lai and Peskin [13] & - & - & 1.447 & 0.165 & 0.3299 \\
\hline
\end{tabular}

\section{References}

[1] Kyoungyoun Kim, Seung-Jin Baek and HyungJin Sung, "An implicit velocity decoupling procedure for the incomp Navier-Stokes equations", Int. J. Numer. Meth. Fluids 2002; 38:125-138 (DOI: 10.1002/fld.205)

[2] J. Kim, D. Kim and H. Choi, "An immersed boundary finite volume method for simulations of flow in complex geometries", J. Compu. Phys. 171 (2001) 132

[3] A.L.F. Lima, A. Silveira-Neto, and J.J.R. Damasceno, "Numerical simulation of two-dimensional flows over a circular cylinder using the immersed boundary method" Journal of Computational Physics 189 (2003) 351-370

[4] Kyoungyoun Kim, Seung-Jin Baek and Hyung Jin Sung, “An implicit velocity decoupling procedure for the incompressible Navier-Stokes equations" Int. J. Numer. Meth. Fluids 2002; 38: 125-138 (DOI: 10.1002/d.205)

[5] Hung Le and Parvez Moin, “An Improvement of Fractional Step method for the Incompressible Navier-Stokes Equations", Journal of Computational Physics 92. 369-379 (1991) 
[6] Choi H, Moin P., "Effects of the computational time step on the numerical solutions of the turbulent flow", J. of Comp Physics; 114: 1-4 (1994)

[7] Perot JB., "An analysis of the fractional step method", Journal of Computational Physics; 108: 51-58 (1993)

[8] S.C. Lo, K.A. Hoffmann, J.F. Dietiker, "Numerical Investigation of High Reynolds Number Flows over Square and Circular Cylinders", Journal of Thermodynamics and Heat Transfer Vol. 19, No. 1, January-March 2005

[9] Wei-Xi Huang and Hyung Jin Sung, "Improvement of mass source/sink for an immersed boundary method" Int. J. Numer. Meth. Fluids; 53: 16591671 (2007)

[10] Ye T, Mittal R., Udaykumar H.S., Shyy W., “An accurate Cartesian grid method for viscous incompressible flows with complex immersed boundaries", Journal of Computational Physics; 156: 209-240 (1999)

[11] Tseng YH, Ferziger J H., "A ghost-cell IBM for flow in complex geometry”, Journal of Computational Physics;192: 593-623 (2003)

[12] Linwei Shen, Eng-Soon Chan, Pengzhi Lin, "Calculation of hydrodynamic forces acting on a submerged moving object using IBM", J. of Computers and Fluids. 2008.07.002, 03/2009; 38(3): 691-702. DOI: 10.1016

[13] Lai and Peskin, "An Immersed Boundary Method with Formal SecondOrder Accuracy and Reduced Numerical Viscosity", J. of Comp Physics 160, 705-719 (2000) 